tolerance was relatively good, despite some severe pulmonary damage probably related to the toxicity of the carmustine.

In view of these results, the BEAM protocol could be used widely in children with relapsed or refractory Hodgkin's lymphoma.

No conflict of interest.

\section{CPC-143 TRABECTEDIN FOR METASTATIC SOFT TISSUE SARCOMA - A RETROSPECTIVE ANALYSIS}

doi:10.1136/ejhpharm-2013-000276.600

AR Gaspar, PF Tavares, J Casanova. Centro Hospitalar e Universitário de Coimbra, UTAL - Bone and Soft Tissue Sarcoma Unit, Coimbra, Portugal

Background Soft tissue sarcomas (STSs) are rare tumours arising from connective tissues characterised by high morphologic and biologic heterogeneity, as well as by limited responsiveness to cytotoxic chemotherapeutic agents. Trabectedin was approved in 2007 for patients with advanced STS after failure of anthracyclines and ifosfamide, or for patients unsuited to receive these agents.

Purpose To obtain basic epidemiological information on patients with soft tissue sarcomas, standard treatment procedures and results of trabectedin treatment in clinical practise.

Materials and Methods This retrospective study analysed 31 STS patients treated with trabectedin between January 2009 and September 2012. A retrospective cohort study of all patients with a diagnosis of STS treated with trabectedin $1.5 \mathrm{mg} / \mathrm{m}^{2}, \mathrm{D} 1,24$ hours' continuous IV infusion, every 3 weeks. Toxicity was evaluated using Common Terminology Criteria for Adverse Events (CTCAE). Progression-free survival curves (PFS) and Overall Survival (a 95\% confidence interval was used) were estimated by using the KaplanMeier method.

Results Median age at the initiation of trabectedin therapy was 52 years (18-79 years).

Leiomyosarcoma was the most frequent tumour (25.8\%) and liposarcoma occurred in $16.2 \%$ of the patients.

Median number of cycles administered was 6.7 ( $2-16$ cycles).

Thrombocytopenia, leukopenia ( $16.1 \%$ of patients), asthenia $(12.9 \%)$ and elevation of liver transaminases ( $9.7 \%$ of patients) were the most frequent adverse effects.

Nine patients achieved a partial remission (PR) and in 3 the disease stabilised (SD).

Median overall survival (95\% CI) was 6.0 months (0.8; 36.1), median progression-free survival (PFS) (95\% CI) was 11.48 months.

PFS for all patients was $90.3 \%$ at three months and $79.0 \%$ at six months.

Conclusions Our results indicate that trabectedin shows promise as an effective and tolerable new drug for the treatment of patients with STS.

No conflict of interest.

\section{CPC-144 TRACING THE RE-EVALUATION OF ANTIBIOTICS AT 48-72 HOURS: IT IS NOT AUTOMATIC...}

doi:10.1136/ejhpharm-2013-000276.601

${ }^{1} \mathrm{G}$ Baussant, ${ }^{2} \mathrm{~A}$ Desante, ${ }^{3} \mathrm{~B}$ Taisne, ' $\mathrm{C}$ Begon Lours. 'Hazebrouck Hospital Center, Pharmacy, Hazebrouck Cedex, France; '2Hazebrouck Hospital Center, Quality, Hazebrouck Cedex, France; ${ }^{3}$ Hazebrouck Hospital Center, Medical unit, Hazebrouck Cedex, France

Background In our hospital, the medication system is totally managed by computers. When physicians sign the computerised prescription, an electronic sheet must be completed for controlled antibiotics. In 2011, pharmacists created a specific second part on the sheet about re-evaluating the antibiotic. Physicians can complete it 72 hours after initiation of empirical treatments as indicated in the recommendations.
Purpose To evaluate the traceability of the re-evaluation of the antibiotic in the paper medical records and in the electronic antibiotics sheets. The results were compared with an audit conducted in 2010 of the re-evaluation in the paper medical records.

Materials and Methods An audit grid was created to assess the traceability of the re-evaluation, the changes of antibiotic treatment after re-evaluation and re-evaluation deadlines.

Results Of 50 medical records audited in the 5 hospital units, 12 were excluded because patients were hospitalised for less than 72 hours. $94.7 \%$ of empirical treatments were re-evaluated, $73.5 \%$ of them before 72 hours ( $84 \%$ in 2010 and $90.7 \%$ of them before 72 hours). Physicians noted the re-evaluation in $58.3 \%$ of paper medical records (38.1\% explicit re-evaluation, $61.9 \%$ implicit) versus $52 \%$ in 2010 (36.4\% explicit re-evaluation, 63.6\% implicit). 100\% of electronic antibiotics sheets were completed: $25 \%$ by physicians and $75 \%$ by the pharmacist after calling the physicians. The re-evaluation led to treatment modification in $41.7 \%$ of the patients: change of the prescribed antibiotic $(33.3 \%)$, change route of administration $(26.7 \%)$, termination of treatment (20\%), adding another antibiotic $(20 \%)$.

Conclusions The rate of re-evaluation on paper medical records wasn't significantly different to the result from a first audit conducted in 2010. Thanks to the pharmacists' involvement, traceability on electronic sheets is being noted correctly. The results will be passed on to the hospital antibiotics committee. Improvements will be proposed for better multidisciplinary collaboration between bacteriologists, pharmacists and physicians.

No conflict of interest.

\section{CPC-145 TREATMENT OF CUTANEOUS CALCIPHYLAXIS WITH SODIUM THIOSULFATE: A CASE REPORT}

doi:10.1136/ejhpharm-2013-000276.602

${ }^{1}$ E Echarri Arrieta, 'A Fernandez-Ferreiro, ${ }^{2} \mathrm{~A}$ Perez-Freiria. ${ }^{1}$ Complejo Hospitalario Universitario de Santiago de Compostela, Servicio de Farmacia, Santiago de Compostela, Spain; ${ }^{2}$ Clinica Souto Boo, Unidad de hemodialisis, Santiago de Compostela, Spain

Background Calciphylaxis is a rare and potentially life-threatening condition. It is thought to result from arterial calcification causing complete vascular occlusion and subsequent cutaneous infarction. Most often, it is a complication of end-stage renal failure or hyperparathyroidism. This condition may be present in up to $4 \%$ of endstage renal disease patients. The clinical picture is typically characterised by very painful skin lesions and ulcerations following calcification and occlusion of small cutaneous arterioles. Recently some evidence supports the use of intravenous sodium thiosulfate (STS) (Hayden M.R. et al, Calciphylaxis: calcific uremic arteriolopathy and the emerging role of sodium thiosulfate, Int Urol Nephrol 2008;40:443-451)

Purpose This abstract focuses on a case report of calciphylaxis successfully resolved with IV STS, as randomised controlled studies on STS efficacy are lacking.

Materials and Methods We report a case of calciphylaxis in a 77-year-old white woman with CKD. The acute presentation was seemingly precipitated by a high calcium-phosphorus product. As the patient was already taking bisphosphonates and phosphate binders, STS was suggested as a good treatment alternative. STS was administered intravenously using $25 \mathrm{~g}$ diluted in $100 \mathrm{cc}$ of normal saline during dialysis.

Results The calciphylaxis episode was related to a high calciumphosphorus product $\left(\mathrm{P}^{*} \mathrm{Ca}=73\right)$, besides a high increase of parathyroid hormone $(800 \mathrm{pg} / \mathrm{ml})$. Clinical signs included cutaneous infarction and pain (photo is included). Four months after the initiation of STS injuries began to improve (photo is included) and the $\mathrm{P}^{*} \mathrm{Ca}$ was reduced but still remained high $\left(\mathrm{P}^{*} \mathrm{Ca}=60\right)$. The parathyroid hormone level continued the same. The patient is still on IV STS treatment. 